\title{
Differential immunogenicity of homologous versus heterologous boost in Ad26.COV2.S vaccine recipients
}

Nicholas Khoo Kim Huat ${ }^{1 *}$, Joey Ming Er Lim ${ }^{1 *}$, Upkar S. Gill ${ }^{2 *}$, Ruklanthi de Alwis ${ }^{1,3}$, Nicole $\operatorname{Tan}^{1}$, Justin Zhen Nan Toh ${ }^{1,3}$, Jane E. Abbott ${ }^{2}$, Carla Usai ${ }^{2}$, Eng Eong Ooi ${ }^{1,3}$, Jenny Guek Hong Low $^{1,4}$, Nina Le Bert ${ }^{1 \#}$, Patrick T. F. Kennedy ${ }^{2 \#}$ \& Antonio Bertoletti ${ }^{1,5 \#}$

*These authors contributed equally

${ }^{*}$ These authors share co-senior authorship

${ }^{1}$ Programme in Emerging Infectious Diseases, Duke-NUS Medical School, Singapore

${ }^{2}$ Barts Liver Centre, Barts and The London School of Medicine \& Dentistry, Queen Mary University of London, London, UK

${ }^{3}$ Viral Research and Experimental Medicine Centre (ViREMiCS), SingHealth Duke-NUS Academic Medical Centre, Singapore

${ }^{4}$ Department of Infectious Diseases, Singapore General Hospital, Singapore

${ }^{5}$ Singapore Immunology Network, A*STAR, Singapore

\section{Corresponding Author:}

Antonio Bertoletti, MD

Programme in Emerging Infectious Diseases

Duke-NUS Medical School

8 College Road

Singapore 169857

Singapore

Tel: +65 92295044

Email: antonio@duke-nus.edu.sg 


\begin{abstract}
Protection offered by COVID-19 vaccines wanes over time, requiring an evaluation of different boosting strategies to revert such a trend and enhance the quantity and quality of Spike-specific humoral and cellular immune responses. These immunological parameters in homologous or heterologous vaccination boosts have thus far been studied for mRNA and ChAdOx1 nCoV-19 vaccines, but knowledge on individuals who received a single dose of Ad26.COV2.S is lacking.

We studied Spike-specific humoral and cellular immunity in Ad26.COV2.S vaccinated individuals ( $n=55$ ) who were either primed with Ad26.COV2.S only ( $n=13$ ), or boosted with a homologous (Ad26.COV2.S, $n=28$ ) or heterologous (BNT162b2, $n=14$ ) second dose. We compared our findings with the results found in individuals vaccinated with a single $(n=16)$ or double $(n=44)$ dose of BNT162b2. We observed that a strategy of heterologous vaccination enhanced the quantity and breadth of both, Spike-specific humoral and cellular immunity in Ad26.COV2.S vaccinated. In contrast, the impact of homologous boost was quantitatively minimal in Ad26.COV2.S vaccinated and Spike-specific antibodies and T cells were narrowly focused to the S1 region. Although a direct association between quantity and quality of immunological parameters and in vivo protection has not been demonstrated, the immunological features of Spike-specific humoral and cellular immune responses support the utilization of a heterologous strategy of vaccine boost in individuals who received Ad26.COV2.S vaccination.
\end{abstract}


medRxiv preprint doi: https://doi.org/10.1101/2021.10.14.21264981; this version posted October 14, 2021. The copyright holder for this preprint (which was not certified by peer review) is the author/funder, who has granted medRxiv a license to display the preprint in perpetuity.

It is made available under a CC-BY-NC-ND 4.0 International license .

\section{Introduction}

Vaccination has been the key strategy to reduce the incidence of SARS-CoV-2 infection and to protect from severe COVID-19 world-wide. Accelerated vaccine development efforts led to the approval of SARS-CoV-2 vaccines utilizing several different technological platforms, that displayed varying clinical efficacy with highest being associated with adenoviral vector and mRNA-based vaccines (1-5). Vaccine-induced protective efficacy is associated with their ability to induce neutralizing anti-Spike antibodies and Spike-specific T cells $(3,6,7)$. Unfortunately, the appearance of the Delta variant and the progressive waning of antibody titers observed over time has reduced the protective efficacy of COVID-19 vaccines (5). These findings have ignited a debate about the need for possible booster vaccinations.

Ad26.COV2 (Johnson \& Johnson) is a single dose vaccine (8) with protective efficacy against severe disease $(4,9)$. Single immunization with Ad26.COV2.S induced rapidly cellular immune responses as well as binding and neutralizing antibodies, including induction of RBD-specific binding antibodies in $90 \%$ of vaccine recipients $(4,9,10)$. A recent report also indicated that vaccination with Ad26.COV2.S leads to persistence of protective efficacy (11). On the flip side, there are other evidences that a single dose of Ad26.COV2.S may not be sufficient; for instance a higher incidence of breakthrough infections with the Delta variant has been observed in comparison to mRNA vaccinated in some U.S. states (12). Additionally, a reduced ability of Ad26.COV2.S to induce antibody responses was reported in immunocompromised individuals (13). As a result, it has been proposed that individuals vaccinated with Ad26.COV2.S should receive a second dose, similarly to the two dose regimen recommended for mRNA-based vaccines (BNT162b2 and mRNA1273) and the adenoviral vector-based vaccine (ChAdOx1 nCov-19).

Vaccine-induced protective efficacy is associated with the ability to induce neutralizing antiSpike antibodies and Spike-specific T cells (14). Data in animal models and in healthy individuals vaccinated with the other adenoviral vector-based vaccine, ChAdOx1 nCov19, followed by BNT162b2 have shown that a heterologous vaccine boost enhances both cellular and humoral immunity (15-18). Data recently reported showed an ability of both homologous and heterologous boost after Ad26.COV2.S to increase Spike-specific antibodies, however a parallel analysis for cellular immunity was not performed (19). 
Therefore, to gain a more comprehensive information on the best boosting strategy in individuals vaccinated with a single dose of Ad26.COV2.S, we studied here, the Spikespecific T and B cell immunogenicity after a homologous or heterologous second vaccination dose. We compared the results with those obtained from individuals vaccinated with a single or double dose of BNT162b2. The quantity and breadth of Spike-specific antibodies and T cells were studied. Collectively, our data show the enhanced immunogenicity of the heterologous boosting strategy in individuals primed with Ad26.COV2.S. 


\section{Methods and Materials}

\section{Subject details}

We recruited 115 study participants who received different vaccination regimes against COVID-19. Details are presented in Table 1.

Peripheral blood mononuclear cell isolation

Peripheral blood of all individuals was collected and peripheral blood mononuclear cells (PBMC) were isolated by Ficoll-Paque density gradient centrifugation.

Peptides

15-mer peptides that are overlapping by 10 amino acids (AA) spanning the entire SARS-CoV2 Spike protein, Nucleoprotein and Membrane protein were synthesized (Genscript) and pooled into 7, 2 and 1 pools of approximately 40 peptides in each pool, respectively $(20,21)$.

\section{SARS-CoV-2 Spike-specific T cell quantification}

The frequency of SARS-CoV-2-specific T cells was quantified as described previously (22). Briefly, cryopreserved PBMCs were stimulated with peptide pools in an IFN- $\gamma$ ELISpot assay. ELISpot plates (Millipore) were coated with human IFN- $\gamma$ antibody overnight at $4^{\circ} \mathrm{C} .400,000$ PBMCs were seeded per well and stimulated for $18 \mathrm{~h}$ with the distinct peptide pools at 2 $\mu \mathrm{g} / \mathrm{ml}$. The plates were then incubated with human biotinylated IFN- $\gamma$ detection antibody, followed by Streptavidin-AP and developed using the KPL BCIP/NBT Phosphatase Substrate. To quantify positive peptide-specific responses, $2 x$ mean spots of the unstimulated wells were subtracted from the peptide-stimulated wells, and the results expressed as spot forming cells $(\mathrm{SFC}) / 10^{6}$ PBMC. Results were excluded if negative control wells had $>30$ $\mathrm{SFC} / 10^{6} \mathrm{PBMC}$ or if positive control wells (PMA/Ionomycin) were negative. 
Surrogate Virus Neutralization Test (sVNT)

The sVNT assay is a proxy measurement of antibodies inhibiting SARS-CoV-2 virus binding to the host cell receptor, human angiotensin-converting enzyme 2 (hACE2), and has been shown to correlate closely with antibody neutralization of SARS-CoV-2 (23). SVNT was measured using a commercial RBD-hACE2 binding inhibition assay called CPASS ${ }^{\mathrm{TM}}$ (GenScript) as per manufacturer guidelines. Briefly, serum was diluted 1:10 in the kit sample buffer, was mixed 1:1 with HRP-conjugated RBD and incubated for 30 mins at $37^{\circ} \mathrm{C}$. RBD-antibody mixtures were then transferred and incubated for 15 mins at $37^{\circ} \mathrm{C}$ in enzyme-linked immunosorbent assay (ELISA) plates coated with recombinant hACE2 receptor. Following incubation, plates were washed with wash solution, incubated with TMB substrate for 12-15 mins and reaction stopped with stop solution. Absorbance was measured at $\mathrm{OD}_{450 \mathrm{~nm}}$. Percent inhibition of RBD-hACE2 binding was computed using the following equation: $\%$ inhibition $=\left[1-\left(\frac{\text { oD with sample }}{O D \text { with Negative Control }}\right)\right] * 100$.

\section{SARS-CoV-2-specific Luminex Antibody assay}

Antigen-specific IgG and IgA responses in serum samples were measured using a previously described bead-based immune-assay with some adjustments (22). Briefly, SARS-CoV-2 recombinant proteins Spike, S1 or S2 (AcroBiosystems) were covalently conjugated to Magpix Luminex beads. Antigen-conjugated beads were then blocked with 1\% BSA (bovine serum albumin, before being probed with either diluted human serum or antibody standards for $1 \mathrm{hr}$ at 37C. Beads were then washed and probed with either anti-human IgGPE (Invitrogen) or anti-human IgA-Biotin (Southern Biotech) followed by Streptavidin-PE (Southern Biotech) for measuring human IgG and IgA, respectively. IgG and IgA binding to antigen were measured as Median Fluorescence Intensity (MFI) using a Magpix instrument (Luminex). MFI values of serum samples were converted to antibody quantity (i.e. $\mathrm{g} / \mathrm{ml}$ ) using anti-Spike IgG and IgA antibody standards (AcroBiosystems). Serum samples were first tested at dilutions 1:100 and 1:2000, if MFI values were above the range of the antibody standards, then serum samples were further diluted to 1:10,000 and tested. 


\section{Detection of SARS-CoV2-specific Memory B cells}

To detect SARS-CoV-2-specific memory B cells, biotinylated protein antigens were individually multimerized with streptavidin (SA) fluorophore conjugates, as described here (24). Briefly, full length Spike protein (RnD Systems) was multimerized with SA-Dylight 550 or SA-Dylight 650 (Thermo Fisher Scientific) in buffer containing 50/50 mixture of 2\% FBS and Brilliant Buffer (BD Bioscience) for 1 hour at $4^{\circ} \mathrm{C}$. Spike protein (RnD Systems) and SADy550/SA-Dy650 were mixed at a 10:1 mass ratio ( 4:1 molar ratio) freshly before every staining. Cells were first stained for 10 minutes at RT with Live/Dead Fixable Blue Stain Reagent (Life Technologies). Subsequently cells were stained with $50 \mu$ l of antigen probe cocktail containing $100 \mathrm{ng}$ of Spike per probe (i.e. 100ng of Spike-Biotin/SA-Dylight 550 and 100ng of Spike-Biotin/SA-Dylight 650) for 1 hour at $4^{\circ} \mathrm{C}$. In parallel, SA-Dylight 550 and SADylight 650 probes (100ng each) not conjugated to protein were used as decoy probes to gate out non-specific streptavidin-binding B cells. Next, cells were stained with an antibody cocktail (against CD3, CD10, CD19, CD21, CD27, CD38, CD40, CD69, CD71, CD95, IgD, IgG, IgM, see Table S1) for 30 mins at $4^{\circ} \mathrm{C}$. Finally, cells were washed and fixed with $1 \%$ formaldehyde before acquisition on a LSR-Fortessa flow cytometer (BD). Analysis of flow cytometry data was performed using FlowJo software, version 10 (BD).

\section{Study approval}

All donors provided written consent. The study was conducted in accordance with the Declaration of Helsinki and approved by the NUS Institutional Review Board (NUS-IRB-2021292), the SingHealth Centralised Institutional Review Board (CIRB ref.: 2018/2387; 2018/3045; 2021/2014) and the Queen Mary University of London Review Board (REC Ref: 20/EE/0154).

\section{Statistical analyses}

All statistical analyses were performed in Prism (GraphPad Software); details are provided in the figure legends. 


\section{Results}

\section{Cohorts of vaccinated individuals}

We studied humoral and cellular immunity in a total of 115 individuals who received different vaccination schedules (Fig. 1A): single dose Ad26.COV2.S (J; $n=13)$, homologous double dose Ad26.COV2.S (J+J; $n=28)$, single dose BNT162b2 (P; $n=16)$, homologous double dose BNT162b2 (P+P; $n=44)$ and heterologous switch dose Ad26.COV2.S followed by BNT162b2 (J+P; $n=14)$. Epidemiological characteristics of the studied population are summarized in Table 1. As controls, we studied 10 to 22 unvaccinated healthy individuals and 40 unvaccinated SARS-CoV-2 convalescents. In the homologous double dose Ad26.COV2.S cohort, the median time between the first and second dose was 56 days (4371 days). In the heterologous switch dose Ad26.COV2.S and BNT162b2 cohort, the median number of days between first and second dose was 31 days, but with a wider range (11-180 days). The time between first and second dose of BNT162b2 was 21 days (21-104 days). Note also that the analysis of humoral and cellular immune parameters was performed at variable intervals after the second dose with a median number of days indicated in Table 1. Humoral responses were characterized by measuring IgG and IgA against the whole Spike, S1 and S2 domains, neutralising antibodies (using the surrogate virus neutralization test; sVNT) and by quantification of Spike-specific memory B cells (Fig. 1B). T cell response was analysed by quantification of IFN- $\gamma$ secreting cells in reaction to peptides covering the whole Spike protein in an ELISpot assay. Seven pools of 15-mer peptides were used to detect Spike-specific T cells, Spike pools 1 to 4 were derived from the S1 chain, including the signal sequence. Spike pools 5 to 7 were derived from the S2 chain (Methods, Fig. 1C).

Furthermore, to ensure that we could differentiate individuals who had prior exposure to SARS-CoV-2 infection, presence of SARS-CoV-2 Membrane and Nucleoprotein-specific T cells were determined (21). 24 individuals tested positive for at least one of the three peptide pools used and they were classified as vaccination in SARS-CoV-2 convalescents in further analyses (Fig. S1).

Quantification of humoral and cellular Spike-specific immune responses 
medRxiv preprint doi: https://doi.org/10.1101/2021.10.14.21264981; this version posted October 14, 2021. The copyright holder for this preprint

We first quantified Spike-specific humoral and cellular immune responses in the five groups of vaccinated naïve individuals (Fig. 2A). Vaccine boost increased the overall profile of humoral immune responses in all individuals irrespective of their first vaccination ( $\mathrm{J}$ or $\mathrm{P})$. In individuals vaccinated with Ad26.COV2.S both homologous $(J+J)$ and heterologous $(J+P)$ vaccination increased the quantity of total Spike IgG and neutralising antibodies (sVNT). However, heterologous $(J+P)$ vaccination induced a higher quantity of anti-Spike IgG and IgA antibodies than homologous $(\mathrm{J}+\mathrm{J}$ ) vaccination (Fig. $2 \mathrm{~A}$ and S2A). The level of neutralising antibodies was also higher in heterologous versus homologous vaccinated individuals even though it did not reach statistical significance. Remarkably, all heterologous vaccinated individuals (9/9) had neutralising antibodies that achieved more than $80 \%$ of inhibition in the sVNT. In contrast, $7 / 21$ of homologous vaccinated $(\mathrm{J}+\mathrm{J})$ had sVNT levels below $50 \%$. Analysis of the frequency of circulating Spike-specific memory B cells was instead poorly indicative of the level of antibodies detected. Many individuals showed no or minimal increase of Spike-specific memory B cell frequency after booster, yet individuals with higher frequency were among the heterologous $(J+P)$ vaccination cohort (Fig. $2 A)$.

The analysis of Spike-specific T cells showed a higher frequency in heterologous $(J+P)$ versus homologous $(\mathrm{J}+\mathrm{J})$ vaccine recipients (347.5 vs $152 \mathrm{SFC} / 10^{6} \mathrm{PBMC}$ ). This was similar to the level observed in double dose BNT161b2 vaccinated individuals. Notably, while Spikespecific T cells were clearly detected in 11/12 individuals vaccinated with a single dose Ad26.COV2.S, 10/23 individuals who received a homologous $(\mathrm{J}+\mathrm{J}$ ) booster displayed a weak level of Spike-specific T cells ( $<30 \mathrm{SFC} / 10^{6} \mathrm{PBMC}$ ) with a frequency similar to that observed in single dose BNT162b2 vaccinated individuals (Fig. 2A).

To ensure that differences in time of sampling after vaccination did not interfere with the observed trends, we investigated the effect of time after vaccination on immunogenicity by plotting sVNT and the frequency of Spike-specific T cells against the day of testing after the last dose of vaccine (Fig. 2B). Overall, as has been reported in previous studies, the Spikespecific $T$ cell frequency and neutralizing antibodies in vaccinated individuals were not significantly reduced over time at least in the first 3-4 months post-vaccination $(20,25)$. Importantly, even though we tested the majority of individuals boosted with heterologous $(J+P)$ vaccine within 30-40 days, Spike IgG quantity and T cell frequency remained high in the individuals tested at day 120 post second dose (Fig. 2B). Moreover, the individuals 
vaccinated with a single dose of Ad26.COV2.S or with homologous boost $(J+J)$ displayed a pattern of Spike IgG and T cells that was not influenced by the time of testing (Fig. 2B). We also analysed Spike-specific T cell frequency in relation to the age and sex of the vaccine recipients (Fig. S3). Undetectable/low frequency of Spike-specific T cells was observed in the homologous vaccinated individuals who were above the age of 50 . However, individuals of similar age (above 50 years old) present in the heterologous (J+P) vaccination cohort, all displayed high frequency of Spike-specific T cells ( $>100$ SFC $/ 10^{6}$ PBMC). Finally, the level of antibodies and $\mathrm{T}$ cell response to the different vaccine regimen were categorized into none/weak, moderate or strong responders based on their percentile ranking (Fig. $2 \mathrm{C}$ ). The heterologous $(J+P)$ vaccination cohort had the highest proportion of strong responders both in antibodies and T cell response. Instead, the homologous $(\mathrm{J}+\mathrm{J})$ group had the highest proportion of weak responders for T cells, lower than in single dose Ad26.COV2.S and equivalent to single dose BNT162b2 vaccinated individuals.

\section{Qualitative analysis of humoral and cellular Spike-specific immune responses}

The ability to produce a polyclonal antibody response targeting different regions of the Spike protein is essential to maintain the protective efficacy of humoral immunity against SARS-CoV-2 variants $(14,26,27)$. Similarly, T cell responses targeting multiple sites of Spike will reduce the chances of viral variants escaping T cell recognition $(14,28)$. Therefore, we analysed qualitative aspects of both humoral and cellular immunity induced by the different vaccination strategies.

First, we analysed the breadth of antibody responses. Antibodies (IgG and IgA) against the S1 (containing RBD) and the S2 regions of Spike were quantified. Homologous or single dose BNT162b2 vaccination elicited antibodies targeting both chains of Spike, while Ad26.COV2.S vaccination mounted an antibody response targeting preferentially the S1 chain (Fig. 3A, $\mathrm{S} 2 \mathrm{~B})$. However, heterologous $(\mathrm{J}+\mathrm{P})$ vaccination appears to broaden the antibody repertoire against Spike since all the heterologous vaccinated individuals (9/9) had antibodies against both domains, while only $2 / 23$ of homologous $(\mathrm{J}+\mathrm{J})$ vaccinated individuals displayed such antibody diversity (Fig. 3B, S1B). 
Also the analysis of the breadth of the Spike-specific $T$ cells confirmed the ability of heterologous vaccination to broaden the immune response against Spike. Figure $3 \mathrm{C}$ shows the frequencies of Spike-specific T cells reactive to the distinct peptide pools covering the different regions of Spike (Fig. 1C). While heterologous (J+P) vaccination expanded a population of T cells able to recognize both S1 and S2 regions of Spike (a pattern similar was observed in the homologous BNT162b2 vaccination group), homologous $(\mathrm{J}+\mathrm{J})$ and single dose $(J)$ vaccination induced T cells primary targeting the $S 1$ chain.

The number of Spike-peptide pools recognised by T cells was also different (Fig. 3D). Individuals on the J+P vaccine regimen had T cells recognizing at least four Spike-specific peptide pools. Furthermore, 8/9 had highly multi-specific $T$ cells recognizing six or seven different peptide pools. In contrast, a homologous Ad26.COV2.S booster did not expand the ability of Spike-specific T cells to recognize different regions (Fig. 3D). Only 4/23 individuals on homologous $\mathrm{J}+\mathrm{J}$ had T cells recognizing at four or more distinct regions of Spike. A similar pattern was observed in single dose Ad26.COV2.S vaccinated individuals. Of concern, 8/23 homologous $(\mathrm{J}+\mathrm{J})$ vaccinated individuals did not develop a $\mathrm{T}$ cell response against any region of Spike (<7.5 SFC $\left./ 10^{6} \mathrm{PBMC}\right)$. In contrast, this was observed only $1 / 12$ single dose Ad26.COV2.S vaccine recipients.

Class switching of Spike-specific memory B cells

High affinity antibodies are produced by memory B cells (MBCs) upon re-encounter with viral antigen $(29,30)$. Particularly, class-switched IgG+ MBCs are responsible for durable humoral responses $(31,32)$. Having observed that a subset of homologous $J+J$ vaccine recipients had none/weak antibody and $\mathrm{T}$ cell responses (Fig. $2 \mathrm{C}$ ), this prompted us to analyse the profile of Spike-specific B cell maturation.

The proportion of class-switched IgG+ Spike-specific MBCs is shown in Figure 4. In all the cohorts we observed a vast heterogeneity in the proportion of class-switched MBCs among the individuals. Both homologous $(J+J ; P+P)$ and heterologous $(J+P)$ booster vaccination increased the proportion of class-switched Spike-specific MBCs in comparison to a single dose of Ad26.COV2.S (J) or BNT162b2 (P) (Fig. 4B). However, there was no significant difference observed between homologous and heterologous boosting (Fig. 4C). 
Characterization of Spike-specific humoral and cellular immune responses in vaccinated SARS-CoV-2 convalescents

Presence of SARS-CoV-2 Membrane and Nucleoprotein-specific T cells allowed us to identify a total of 23 individuals who were vaccinated with different regimens but were likely infected by SARS-CoV-2 before or during the vaccination regimens (21). The limited number of these individuals categorized within the different vaccination strategies did not allow us to perform statistically significant comparisons. Note, that in the cohort of single dose Ad26.COV2.S (J) there was only one convalescent, hence any measurement of "possible boosting effect" by the second dose cannot be performed. Nevertheless, we compared the quantity of antibodies (total anti-Spike IgG, neutralizing antibodies SVNT) and Spike-specific T cells induced by the different vaccination regime in naïve versus convalescent individuals (Fig. 5A). We observed that all convalescent individuals elicited a stronger humoral and cellular immunity than naïve individuals irrespective of the vaccination regime (Fig. 5A), in line with recent data (33-35).

In addition, the different vaccination strategies in convalescent individuals boosted neutralizing antibodies above $90 \%$ inhibition, in comparison to the $50 \%$ inhibition present in non-vaccinated convalescents 1 year post infection. In contrast, their efficacy in boosting cellular immunity was minimal. The frequency of Spike-specific $T$ cells was marginally increased $(J+J)$ or even lower $(P$ and $P+P)$ than their level detected in unvaccinated convalescent individuals (Fig. 5B). However, high frequency of Spike-specific $T$ cells was detected in heterologous vaccinated individuals. $3 / 5$ individuals in this group displayed a quantity of Spike-specific T cells exceeding $1000 \mathrm{SFC} / 10^{6} \mathrm{PBMC}$. 


\section{Discussion}

Our study provides information on how to boost Spike-specific humoral and cellular immunity in individuals vaccinated with a single dose of Ad26.COV2.S.

Heterologous booster vaccination with a dose of BNT162b2 resulted in elevated titers of anti-Spike IgG and neutralizing antibodies and high frequency of Spike-specific T cell in all the individuals tested.

In addition, heterologous vaccination expanded the breadth of both humoral and $\mathrm{T}$ cell immunity. The results of Spike-specific T cells were particularly robust since we observed that $8 / 9$ of the heterologous vaccinated individuals possessed $T$ cells widely scattered among the whole length of the Spike protein. The immunological correlates of protection induced by the vaccines are still only hypothesized (36). Thus, we cannot conclude that the enhanced immunogenicity of heterologous vaccination will translate in a superior protective efficacy. However, it is likely that future prospective trials of vaccine efficacy will confirm that quantity and quality of humoral and cellular immunity might directly translate into protective efficacy against SARS-CoV-2 infection and COVID-19 disease.

Our observation that a heterologous vaccination strategy in Ad26.COV2.S vaccinated individuals is more immunogenic than a homologues boost was also confirmed in convalescents and is in line with the results obtained in individuals vaccinated with ChadOx1 nCov-19, another adenoviral-based vaccine. Heterologous vaccination after a single dose of ChadOx1 nCov-19 was recommended in several countries due to the problem of intermittent supply and of rare thrombotic events associated with ChadOx1 nCov-19 (37). Analysis of immunogenicity in individuals boosted with mRNA-based vaccines after a single dose of ChadOx1 nCov-19 revealed an enhanced quantitative profile of antibody and $\mathrm{T}$ cell response compared to homologous booster vaccination $(15,16)$. The superior immunogenicity of heterologous vaccination with a combination of vaccines utilizing different expression vectors (38) has also been seen in other vaccination strategies against different viruses (EBOLA, HIV, HBV (39-41)) and other pathogens (malaria, TB $(42,43)$ ). This further suggests that heterologous prime-boost vaccination strategies involving different types of vaccine should be considered. For instance, using mRNA-based followed by an adenoviral-vector based vaccine might be able to delay the reduction of anti-Spike humoral immunity observed after homologous mRNA vaccination (5). 
medRxiv preprint doi: https://doi.org/10.1101/2021.10.14.21264981; this version posted October 14, 2021. The copyright holder for this preprint (which was not certified by peer review) is the author/funder, who has granted medRxiv a license to display the preprint in perpetuity.

It is made available under a CC-BY-NC-ND 4.0 International license .

Importantly, although we observed that homologous vaccination with Ad26.COV2.S enhances the quantity of antibody production, only heterologous boosting expanded the ability of antibodies to recognise the S2 region of Spike. In addition, a second dose of Ad26.COV2.S did not enhance the quantity and breadth of Spike-specific $T$ cells, and appeared detrimental in some individuals. More than one third (8/23) of individuals boosted with homologous Ad26.COV2.S vaccine did not display detectable Spike-specific T cell responses. This proportion of individuals with a very week T cell response was higher than in individuals who received a single dose of Ad26.COV2.S (1/12).

The inability of a homologous second dose Ad26.COV2.S to boost cellular immunity was also observed in non-human primates (44) as well as in individuals receiving homologous ChadOx1 nCov-19 vaccination $(16,17)$. However, the low level of Spike-specific T cells detected here in the individuals with Ad26.COV2.S homologous boost was unexpected and call for a careful evaluation of such strategy in individuals who already received a single dose of Ad26.COV2.S. In animals vaccinated with two doses of Ad26.COV2.S, the Spikespecific T cell response was found to be more stable (44). A parallel analysis of both humoral and cellular immune parameters in larger group of Ad26.COV2.S vaccinated individuals that are followed longitudinally is needed.

The discrepancy between levels of Spike-specific humoral and cellular immune responses has been also frequently observed in SARS-CoV-2 convalescents and vaccine recipients (reviewed in (45)). If we exclude the early phases of convalescence and vaccination, the level of Spike-specific antibodies and T cells appears independently regulated in numerous studies $(20,25,46)$. As such, the data gathered here further support the concept that immunogenicity of vaccines should be comprehensively evaluated in its cellular and humoral branch. Humoral analysis alone cannot be used to evaluate the induction of virusspecific T cells.

There are some important limitations in our study. In addition to the small sample size, the cross-sectional nature of the study did not allow to precisely evaluate the modification of Spike-specific T cell frequency induced by the second dose at individual level. Furthermore, the analysis of both humoral and cellular immunity was performed at different time points after vaccination. Even though we showed that the level of Spike-specific $T$ cells was minimally reduced within the first 6 months after vaccination and did not appear to 
influence the different pattern of Spike-specific T cells, an analysis at identical time points after boosting is indicated to demonstrate the enhanced immunogenicity of heterologous vaccination and its durability over time. Finally, the limited quantity of PBMCs collected only allowed us to perform a simple ELISpot analysis of the T cell response, a method that cannot discriminate whether the Spike-specific T cells induced by different vaccination regimens are CD4 or CD8 T cells. Such information would be needed to better characterize the possible further qualitative differences in T cells responses induced by the different vaccination regimens.

In conclusion, while the Ad26.COV2.S vaccine has been initially proposed as a single dose vaccine, the progressive reduction of its protective efficacy against SARS-CoV-2 infection over time have warranted a better definition of the best boosting strategy (12). Here we provide data that demonstrate the enhanced immunogenicity of heterologous versus homologous boost vaccination in Ad26.COV2.S vaccine recipients. Despite representing a minority within the vaccinated individuals worldwide, the Ad26.COV2.S vaccine recipients deserve information that can guide their future vaccination choice.

\section{Declaration of interest}

N. Le Bert and A. Bertoletti reported a patent for a method to monitor SARS-CoV-2-specific T cells in biological samples pending. The other authors have declared that no conflict of interest exists

\section{Acknowledgments}

We would like to thank all clinical and nursing staff who provided care to all vaccinated individuals and aided in co-ordinating collection of the samples. We are also grateful to all clinical trial coordinators and staff at Barts Vaccine centre for assistance in recruitment organisation of vaccine recipients.

\section{Funding source}


This study is partially supported by the Singapore Ministry of Health's National Medical Research Council under its COVID-19 Research Fund (COVID19RF3-0060, COVID19RF-001 and COVID19RF-008), The Medical College St. Bartholomew's Hospital Trustees - Pump Priming Fund for SMD COVID-19 Research. In addition, USG is supported by grant funding from Academy of Medical Sciences Starter Grant (SGL021/1030), Seedcorn funding Rosetrees/Stoneygate Trust (A2903) and Early Career Research Award from The Medical Research Foundation (MRF-044-0004-F-GILL-C0823). PTFK is supported by funding from Barts Charity Project Grants (723/1795 and MGU/0406) and an NIHR Research for patient benefit award (PB-PG-0614-34087). 


\section{References}

1. Voysey $M$ et al. Safety and efficacy of the ChAdOx1 nCoV-19 vaccine (AZD1222) against SARS-CoV-2: an interim analysis of four randomised controlled trials in Brazil, South Africa, and the UK. Lancet 2021;397(10269):99-111.

2. Folegatti PM et al. Safety and immunogenicity of the ChAdOx1 nCoV-19 vaccine against SARS-CoV-2: a preliminary report of a phase $1 / 2$, single-blind, randomised controlled trial. Lancet 2020;396(10249):467-478.

3. Polack FP et al. Safety and Efficacy of the BNT162b2 mRNA Covid-19 Vaccine. N. Engl. J. Med. 2020;383(27):2603-2615.

4. Sadoff J et al. Safety and Efficacy of Single-Dose Ad26.COV2.S Vaccine against Covid-19. N. Engl. J. Med. 2021;384(23):2187-2201.

5. Chemaitelly $\mathrm{H}$ et al. Waning of BNT162b2 Vaccine Protection against SARS-CoV-2 Infection in Qatar. N. Engl. J. Med. 2021;1-15.

6. Sahin $U$ et al. BNT162b2 vaccine induces neutralizing antibodies and poly-specific T cells in humans. Nature 2021;595(7868):572-577.

7. Oberhardt $V$ et al. Rapid and stable mobilization of CD8+ T cells by SARS-CoV-2 mRNA vaccine. Nature 2021;597(7875):268-273.

8. Bos R et al. Ad26 vector-based COVID-19 vaccine encoding a prefusion-stabilized SARSCoV-2 Spike immunogen induces potent humoral and cellular immune responses. npj Vaccines 2020;5(1):1-11.

9. Alter $\mathrm{G}$ et al. Immunogenicity of Ad26.COV2.S vaccine against SARS-CoV-2 variants in humans. Nature 2021;596(7871):268-272.

10. Stephenson KE et al. Immunogenicity of the Ad26.COV2.S Vaccine for COVID-19. JAMA $2021 ; 325(15): 1535$.

11. Polinski JM et al. Effectiveness of the Single-Dose Ad26.COV2.S COVID Vaccine [preprint]. https://doi.org/10.1101/2021.09.10.21263385. Posted on medRxiv September 16, 2021.

12. Brown CM et al. Outbreak of SARS-CoV-2 Infections, Including COVID-19 Vaccine 
Breakthrough Infections, Associated with Large Public Gatherings - Barnstable County, Massachusetts, July 2021. MMWR. Morb. Mortal. Wkly. Rep. 2021;70(31):1059-1062.

13. Garcia P et al. COVID19 vaccine type and humoral immune response in patients receiving dialysis [preprint]. https://doi.org/10.1101/2021.08.02.21261516. Posted on medRxiv August 04, 2021.

14. Cevik M, Grubaugh ND, Iwasaki A, Openshaw P. COVID-19 vaccines]: Keeping pace with SARS-CoV-2 variants. Cell 2021;184(20):5077-5081.

15. Schmidt $\mathrm{T}$ et al. Immunogenicity and reactogenicity of heterologous ChAdOx1 nCoV19/mRNA vaccination. Nat. Med. [published online ahead of print: 2021]; doi:10.1038/s41591-021-01464-w

16. Barros-Martins J et al. Immune responses against SARS-CoV-2 variants after heterologous and homologous ChAdOx1 nCoV-19/BNT162b2 vaccination. Nat. Med. 2021;27(9):1525-1529.

17. Hillus $D$ et al. Safety, reactogenicity, and immunogenicity of homologous and heterologous prime-boost immunisation with ChAdOx1 nCoV-19 and BNT162b2: a prospective cohort study. Lancet Respir. Med. 2021;2600(21):1-4.

18. Borobia AM et al. Immunogenicity and reactogenicity of BNT162b2 booster in ChAdOx1S-primed participants (CombiVacS): a multicentre, open-label, randomised, controlled, phase 2 trial. Lancet 2021;398(10295):121-130.

19. Atmar RL et al. Heterologous SARS-CoV-2 Booster Vaccinations: Preliminary Report [preprint]. https://doi.org/10.1101/2021.10.10.21264827. Posted on medRxiv October 13, 2021.

20. Tan AT et al. Rapid measurement of SARS-CoV-2 spike T cells in whole blood from vaccinated and naturally infected individuals. J. Clin. Invest. 2021;131(17):2021.06.29.450293.

21. Le Bert $\mathrm{N}$ et al. Highly functional virus-specific cellular immune response in asymptomatic SARS-CoV-2 infection. J. Exp. Med. 2021;218(5). doi:10.1084/JEM.20202617

22. Kalimuddin $\mathrm{S}$ et al. Early $\mathrm{T}$ cell and binding antibody responses are associated with COVID-19 RNA vaccine efficacy onset. Med 2021;2(6):682-688.e4. 
23. Tan CW et al. A SARS-CoV-2 surrogate virus neutralization test based on antibodymediated blockage of ACE2-spike protein-protein interaction. Nat. Biotechnol. 2020;38(9):1073-1078.

24. Goel RR et al. Distinct antibody and memory B cell responses in SARSCoV-2 naïve and recovered individuals following mRNA vaccination. Sci. Immunol. 2021;6(58):1-19.

25. Dan JM et al. Immunological memory to SARS-CoV-2 assessed for up to 8 months after infection. Science. 2021;371(6529). doi:10.1126/science.abf4063

26. Chen RE et al. Resistance of SARS-CoV-2 variants to neutralization by monoclonal and serum-derived polyclonal antibodies. Nat. Med. 2021;27(4):717-726.

27. Garcia-Beltran WF et al. Multiple SARS-CoV-2 variants escape neutralization by vaccineinduced humoral immunity. Cell 2021;184(9):2372-2383.e9.

28. Tarke $A$ et al. Comprehensive analysis of $T$ cell immunodominance and immunoprevalence of SARS-CoV-2 epitopes in COVID-19 cases. Cell Reports Med. 2021;2(2):100204.

29. Kurosaki T, Kometani K, Ise W. Memory B cells. Nat. Rev. Immunol. 2015;15(3):149-159.

30. Palm AKE, Henry C. Remembrance of Things Past: Long-Term B Cell Memory After Infection and Vaccination. Front. Immunol. 2019;10(July):1787.

31. Amanna IJ, Carlson NE, Slifka MK. Duration of Humoral Immunity to Common Viral and Vaccine Antigens. N. Engl. J. Med. 2007;357(19):1903-1915.

32. Ogega CO et al. Durable SARS-CoV-2 B cell immunity after mild or severe disease. J. Clin. Invest. 2021;131(7). doi:10.1172/JCl145516

33. Reynolds $\mathrm{CJ}$ et al. Prior SARS-CoV-2 infection rescues $\mathrm{B}$ and $\mathrm{T}$ cell responses to variants after first vaccine dose. Science. 2021;372(6549):1418-1423.

34. Prendecki $M$ et al. Effect of previous SARS-CoV-2 infection on humoral and T-cell responses to single-dose BNT162b2 vaccine. Lancet 2021;397(10280):1178-1181.

35. Lozano-Ojalvo D et al. Differential effects of the second SARS-CoV-2 mRNA vaccine dose on T cell immunity in naive and COVID-19 recovered individuals. Cell Rep. 2021;36(8). doi:10.1016/j.celrep.2021.109570 
36. Khoury DS et al. Neutralizing antibody levels are highly predictive of immune protection from symptomatic SARS-CoV-2 infection. Nat. Med. 2021;27(7):1205-1211.

37. ECDC. Overview of EU/EEA country recommendations on COVID-19 vaccination with Vaxzevria, and a scoping review of evidence to guide decision-making. https://www.ecdc.europa.eu/en/publications-data/overview-eueea-countryrecommendations-covid-19-vaccination-vaxzevria-and-scoping. cited October 13, 2021

38. Woodland DL. Jump-starting the immune system: Prime-boosting comes of age. Trends Immunol. 2004;25(2):98-104.

39. Backes $S$ et al. Protein-prime/modified vaccinia virus Ankara vector-boost vaccination overcomes tolerance in high-antigenemic HBV-transgenic mice. Vaccine 2016;34(7):923932.

40. Anywaine $Z$ et al. Safety and Immunogenicity of a 2-Dose Heterologous Vaccination Regimen with Ad26.ZEBOV and MVA-BN-Filo Ebola Vaccines: 12-Month Data from a Phase 1 Randomized Clinical Trial in Uganda and Tanzania. J. Infect. Dis. 2019;220(1):46-56.

41. Baden LR et al. Safety and immunogenicity of two heterologous HIV vaccine regimens in healthy, HIV-uninfected adults (TRAVERSE): a randomised, parallel-group, placebocontrolled, double-blind, phase 1/2a study. Lancet HIV 2020;7(10):e688-e698.

42. Schneider J et al. Enhanced immunogenicity for CD8+ T cell induction and complete protective efficacy of malaria DNA vaccination by boosting with modified vaccinia virus Ankara. Nat. Med. 1998;4(4):397-402.

43. McShane $\mathrm{H}$, Hill A. Prime-boost immunisation strategies for tuberculosis. Microbes Infect. 2005;7(5-6):962-967.

44. Solforosi L et al. Immunogenicity and efficacy of one and two doses of Ad26 . COV2 . S COVID vaccine in adult and aged NHP2021;218(7).

45. Bertoletti A, Le Bert N, Qui M, Tan AT. SARS-CoV-2-specific T cells in infection and vaccination. Cell. Mol. Immunol. 2021;(July):1-6.

46. Chia WN et al. Dynamics of SARS-CoV-2 neutralising antibody responses and duration of immunity: a longitudinal study. The Lancet Microbe 2021;2(6):e240-e249. 
medRxiv preprint doi: https://doi.org/10.1101/2021.10.14.21264981; this version posted October 14, 2021. The copyright holder for this preprint (which was not certified by peer review) is the author/funder, who has granted medRxiv a license to display the preprint in perpetuity.

\section{It is made available under a CC-BY-NC-ND 4.0 International license .}

Table 1: Demographics of vaccinated individuals

\begin{tabular}{|c|c|c|c|c|c|c|}
\hline Characteristics & $\begin{array}{c}\mathrm{J} \\
\text { (Ad26.cov2.S) } \\
\text { (single dose) }\end{array}$ & $\begin{array}{c}\mathrm{J}+\mathrm{J} \\
\text { (Ad26.COV2.S) } \\
\text { (double dose) }\end{array}$ & $\begin{array}{c}P \\
\text { (BNT162b2) } \\
\text { (single dose) }\end{array}$ & $\begin{array}{c}\text { P+P } \\
\text { (BNT162b2) } \\
\text { (double dose) }\end{array}$ & $\begin{array}{c}\mathrm{J}+\mathrm{P} \\
\text { (Ad26.COV2.S } \\
\text { \& BNT162b2) }\end{array}$ & Total \\
\hline No. of participants & 13 & 28 & 16 & 44 & 14 & 115 \\
\hline \multicolumn{7}{|l|}{ Sex - no. (\%) } \\
\hline Male & $6(46.2)$ & $18(64.2)$ & $7(43.7)$ & $15(34.1)$ & $9(64.3)$ & $55(47.8)$ \\
\hline Female & $7(53.8)$ & $10(35.8)$ & $9(56.3)$ & $29(65.9)$ & $5(35.7)$ & $60(52.1)$ \\
\hline \multicolumn{7}{|l|}{$\begin{array}{c}\text { Infection status - no. } \\
(\%)\end{array}$} \\
\hline Naïve & $12(92.3)$ & $23(82.1)$ & $10(62.5)$ & $37(84.1)$ & $9(64.3)$ & $91(79.1)$ \\
\hline Infected & $1(7.7)$ & $5(17.9)$ & $6(37.5)$ & $7(15)$. & $5(35.7)$ & $24(20.9)$ \\
\hline \multicolumn{7}{|l|}{ Age - yr } \\
\hline Mean & 39.2 & 51.2 & 42.8 & 40.6 & 45.5 & 44.2 \\
\hline Range & $25-69$ & $25-75$ & $23-53$ & $23-60$ & $25-70$ & $23-75$ \\
\hline \multicolumn{7}{|l|}{$\begin{array}{c}\text { Days between prime } \\
\text { and boost }\end{array}$} \\
\hline Median & - & 56 & - & 21 & 31 & - \\
\hline Range & - & $43-70$ & - & 21-104 & $11-180$ & - \\
\hline \multicolumn{7}{|l|}{ Days post last dose } \\
\hline Median & 80 & 49.5 & 60 & 94 & 32 & 77.5 \\
\hline Range & $23-169$ & $21-164$ & $22-78$ & $21-151$ & $21-124$ & $7-169$ \\
\hline \multicolumn{7}{|l|}{$\begin{array}{c}\text { Race or ethnic group - } \\
\text { no. }(\%)\end{array}$} \\
\hline $\begin{array}{c}\text { White } \\
\text { (Other/British/Irish) }\end{array}$ & $11(84.6)$ & $21(75)$ & $8(50)$ & $5(11.4)$ & $11(75.6)$ & $56(48.7)$ \\
\hline Black & $1(7.6)$ & - & - & - & - & $1(0.9)$ \\
\hline $\begin{array}{l}\text { Asian or South Asian } \\
\text { (Indian/Chinese/Other) }\end{array}$ & $1(7.6)$ & $4(14.3)$ & $6(37.5)$ & $36(81.8)$ & $2(14.3)$ & $49(42.6)$ \\
\hline $\begin{array}{c}\text { Caucasian / Mixed / } \\
\text { Others }\end{array}$ & - & $2(7.2)$ & $1(6.2)$ & $3(6.8)$ & $1(7.1)$ & $7(6.1)$ \\
\hline Unknown/Undisclosed & - & $1(3.5)$ & $1(6.2)$ & - & - & $2(1.7)$ \\
\hline
\end{tabular}


medRxiv preprint doi: https://doi.org/10.1101/2021.10.14.21264981; this version posted October 14, 2021. The copyright holder for this preprint (which was not certified by peer review) is the author/funder, who has granted medRxiv a license to display the preprint in perpetuity. It is made available under a CC-BY-NC-ND 4.0 International license.

Supplementary Table 1: List of antibodies used for Spike-specific B cell phenotyping

\begin{tabular}{|c|c|c|}
\hline Antibody & Catalogue Number & Concentration \\
\hline Brilliant Violet $605^{\mathrm{TM}}$ anti-human CD3 Antibody & 317322 & 2:50 \\
\hline PE-CF594 Mouse Anti-Human CD10 & 562396 & $2: 50$ \\
\hline BV510 Mouse Anti-Human CD19 & 562947 & $2: 50$ \\
\hline BV421 Mouse Anti-Human CD21 & 562966 & $1: 50$ \\
\hline BV650 Mouse Anti-Human CD27 & 563228 & $3: 50$ \\
\hline PE/Cyanine7 anti-human CD38 Antibody & 356608 & $2: 50$ \\
\hline PerCP/Cyanine5.5 anti-human CD40 Antibody & 334316 & $2: 50$ \\
\hline FITC anti-human CD71 Antibody & 334104 & $3: 50$ \\
\hline APC-Cy ${ }^{\mathrm{TM}} 7$ Mouse Anti-Human CD69 & 557756 & $1: 50$ \\
\hline BUV737 Mouse Anti-Human CD95 & 564710 & $1: 50$ \\
\hline Alexa Fluor ${ }^{\circledast} 700$ anti-human IgD Antibody & 348230 & $2: 50$ \\
\hline BV786 Mouse Anti-Human IgG & 564230 & $2: 50$ \\
\hline Brilliant Violet $711^{\mathrm{TM}}$ anti-human IgM Antibody & 314540 & $2: 50$ \\
\hline LIVE/DEAD ${ }^{\mathrm{TM}}$ Fixable Blue Dead & L23105 & $2: 1000$ \\
\hline
\end{tabular}




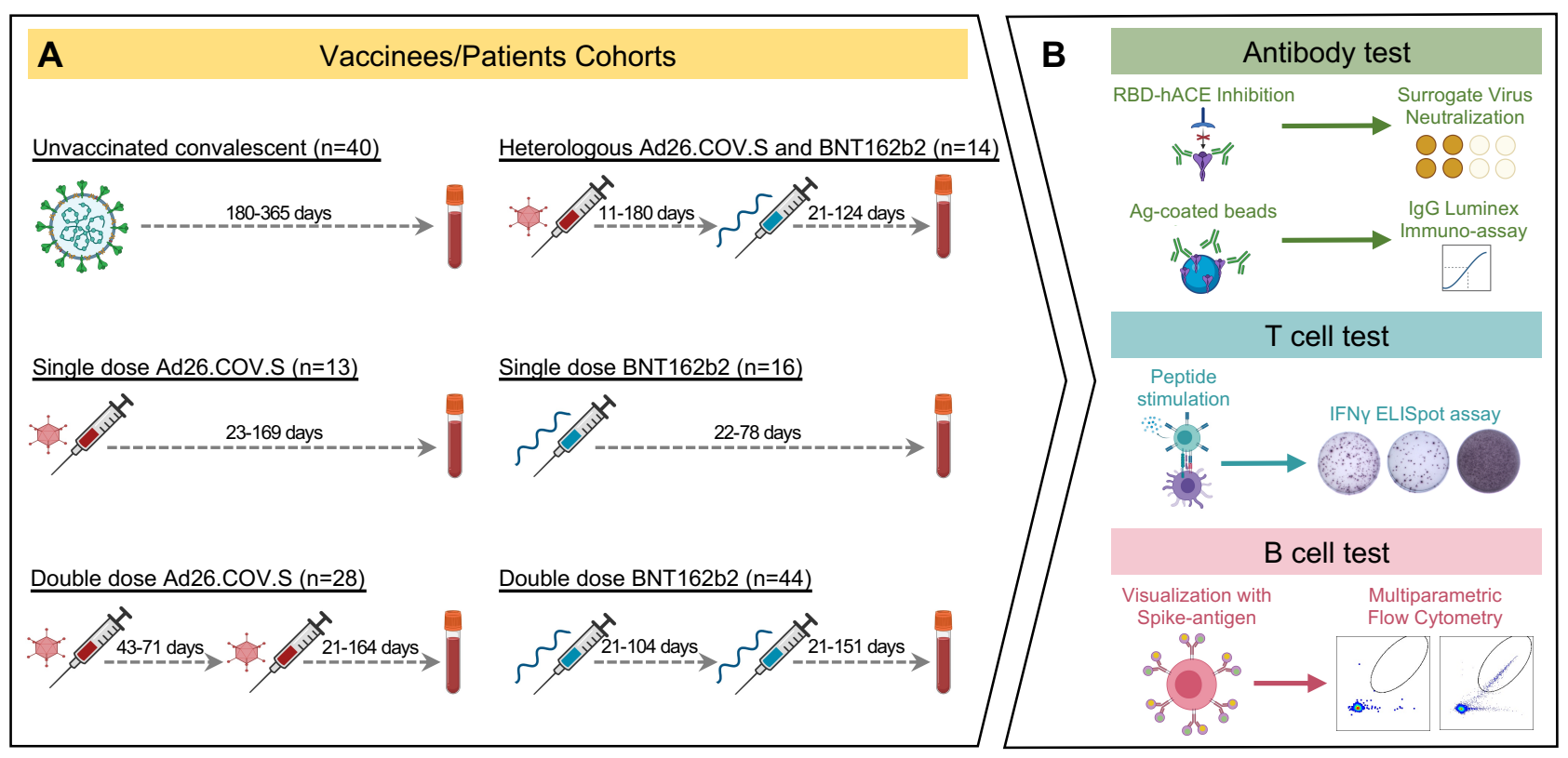

C

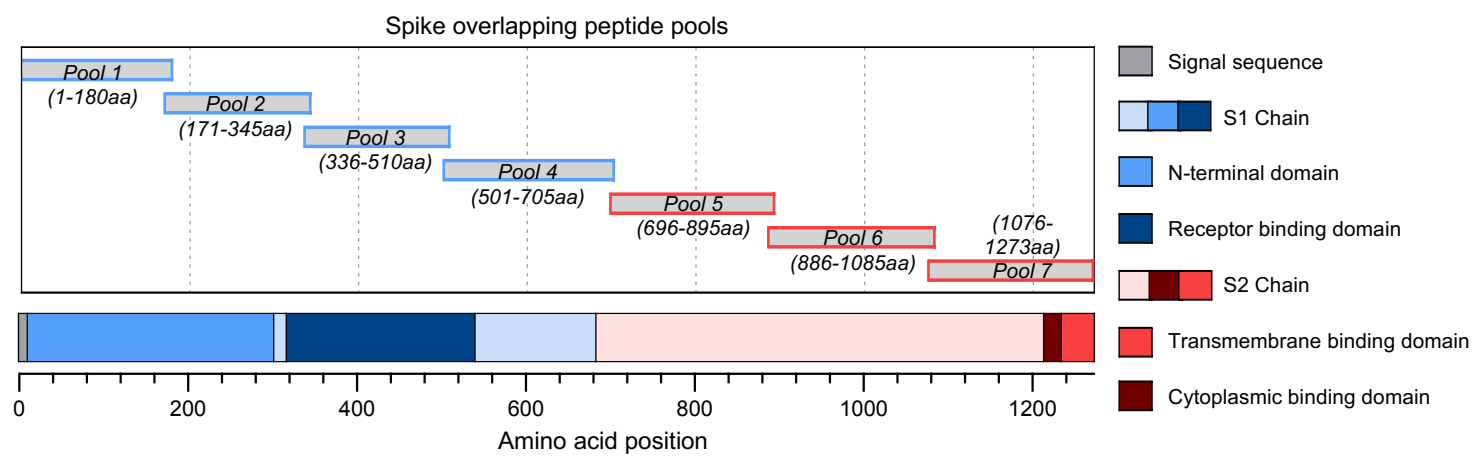

\section{Figure 1: Vaccinated individuals and immune characterization}

(A) Immune characterization was done in six cohorts: single dose Ad26.COV2.S (J), double dose Ad26.COV2.S (J+J), heterologous Ad26.COV2.S + BNT162b2 (J+P), single dose BNT162b2 (P) and double dose BNT162b2 (P+P). Unvaccinated cohort was used as a control (Unvac). Thenumber of individuals for each cohort, the interval between first and second dose of vaccine and the range of days for blood collection after last dose of vaccine are indicated. Sampling for immune analysis (humoral and cellular) was done only after the last vaccination dose both in homologous and heterologous vaccinated groups. (B) Schematic of humoral and cellular analysis. (C) Schematic representation of the location of the seven Spike-specific peptide pools containing 15-mer overlapping peptides spanning the entire Spike protein. Pools 1 to 4 contain peptides from the signal peptide and the S1 chain. Pools 5 to 7 encompass the $\mathrm{S} 2$ chain together with the transmembrane and cytoplasmic domains. 


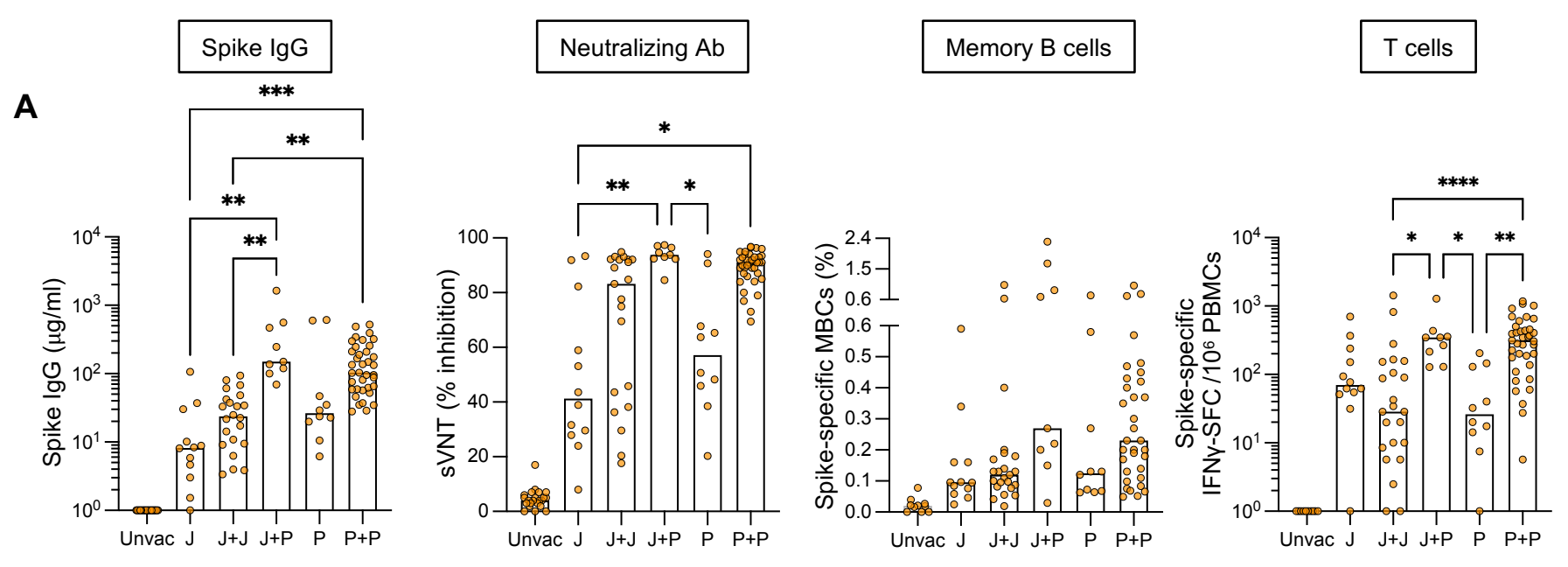

B
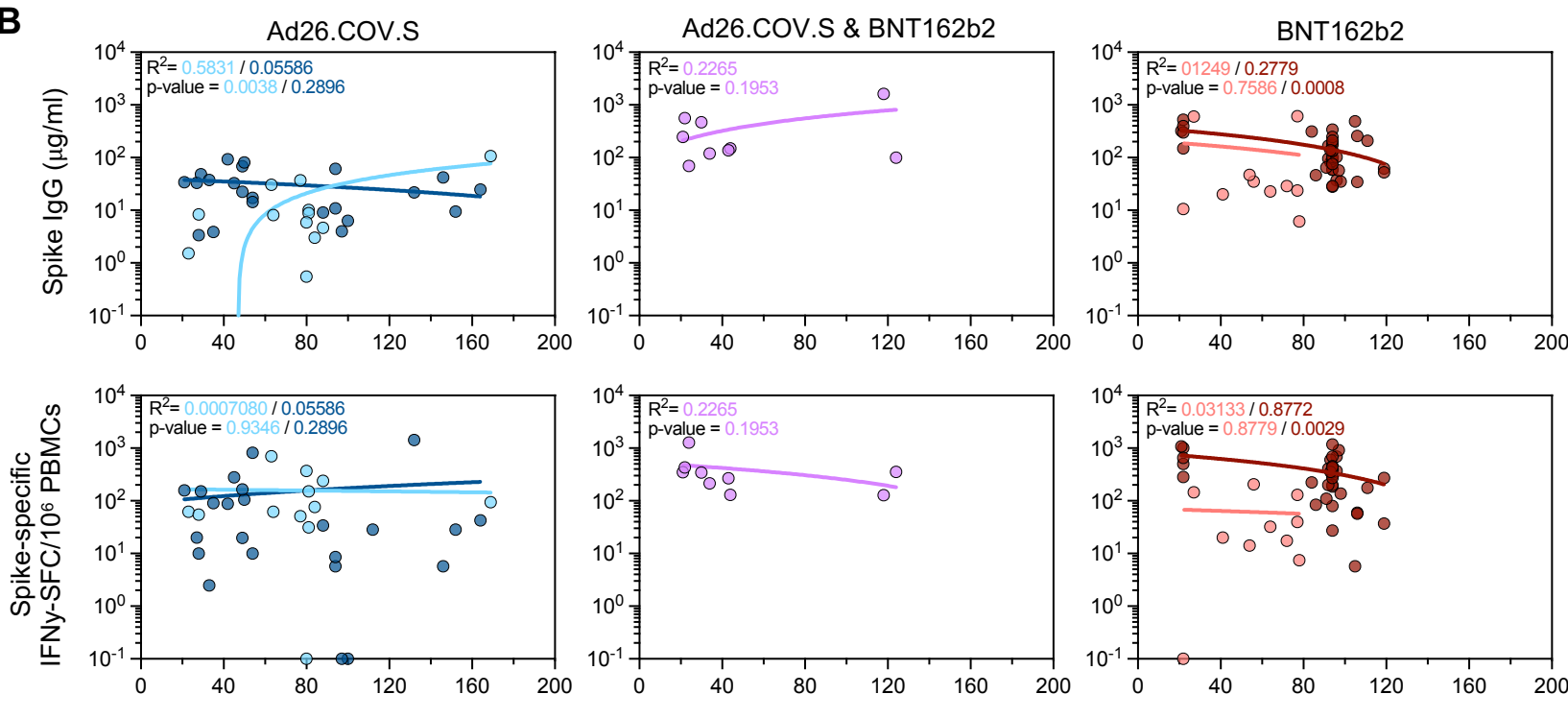

Days from last dose of vaccine

C
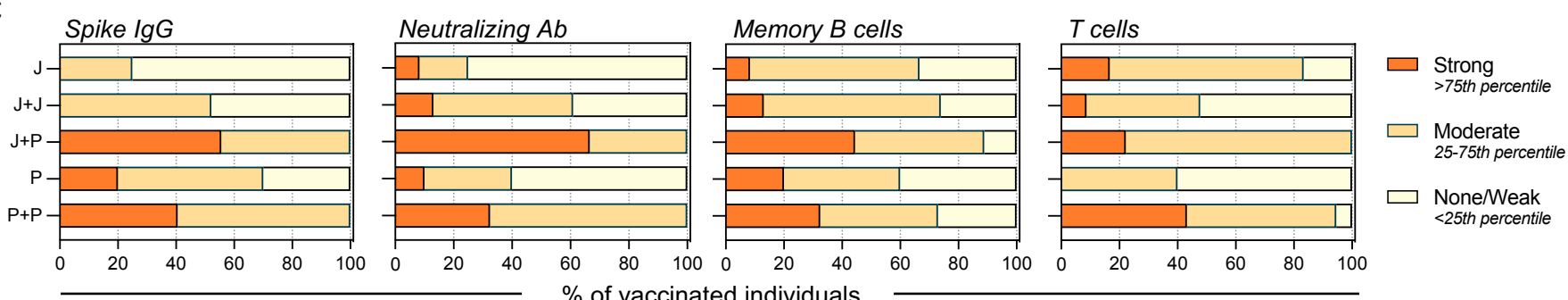

\section{Figure 2: Quantification of humoral and cellular Spike-specific immune responses}

(A) Spike IgG antibody, sVNT, Spike-specific MBCs and T cell responses were tested in five cohorts of naïve vaccinated individuals: J $(n=12), J+J(n=23), J+P(n=9), P(n=10)$ and $P+P(n=37)$. A naïve unvaccinated cohort was used as a control, Unvac $(n=10-22)$. Bars denote the median value of each group. Each dot represents an individual. Significant differences in each group were analysed by one-way ANOVA and the adjusted $p$-value (adjusted for multiple comparison) are shown. No significance is not shown, ${ }^{*}=\mathrm{P} \leq 0.05 ; * *=\mathrm{P} \leq 0.01 ; * * *=\mathrm{P} \leq 0.001 ; * * * *=\mathrm{P} \leq 0.0001$. (B) Linear regression analysis between neutralizing antibody activity (top panels) or Spike-specific $T$ cell frequency (bottom panels) and time of testing after last vaccine dose (days). Goodness of fit and p-value ar4e shown in plots. (C) Bar graphs show the proportion of vaccinees with varying levels of Spike-IgG antibodies, neutralizing antibodies, Spike-specific B and T cell frequencies. The type of responders (Strong/moderate/none-weak) was expressed as a fraction of the number of vaccine recipients in each cohort. Type of responders were determined by percentile score calculated from all the vaccinees $(n=87-91)$. 
A
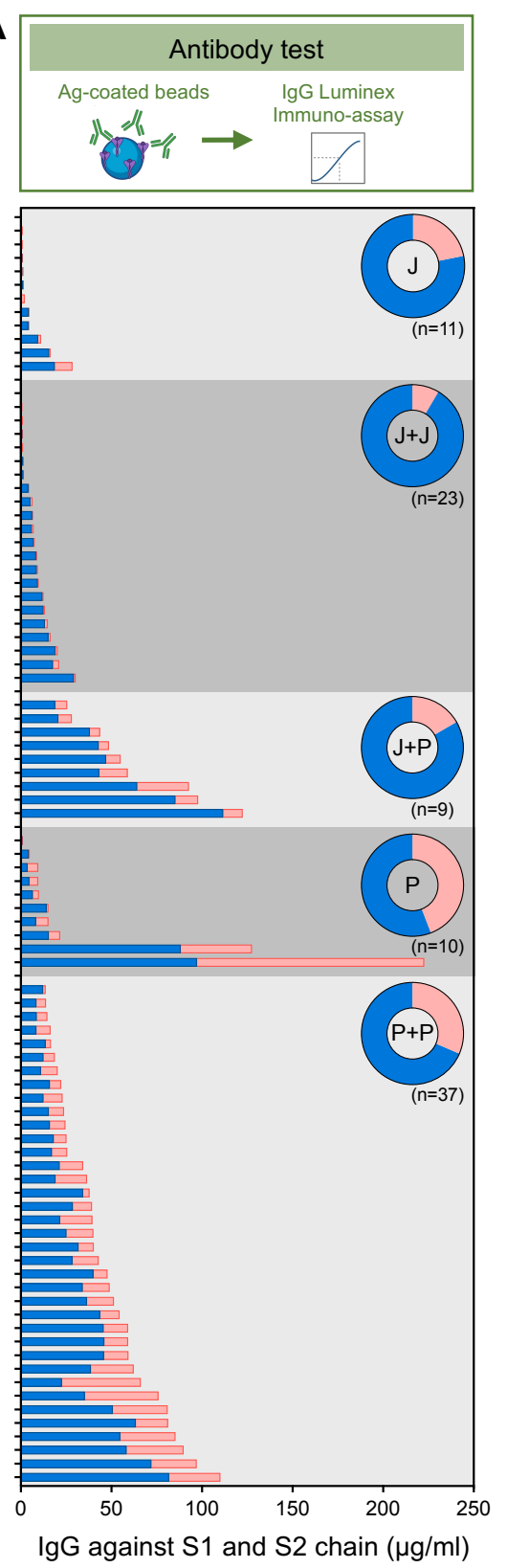

S1 chain $\square$ S2 chain
B

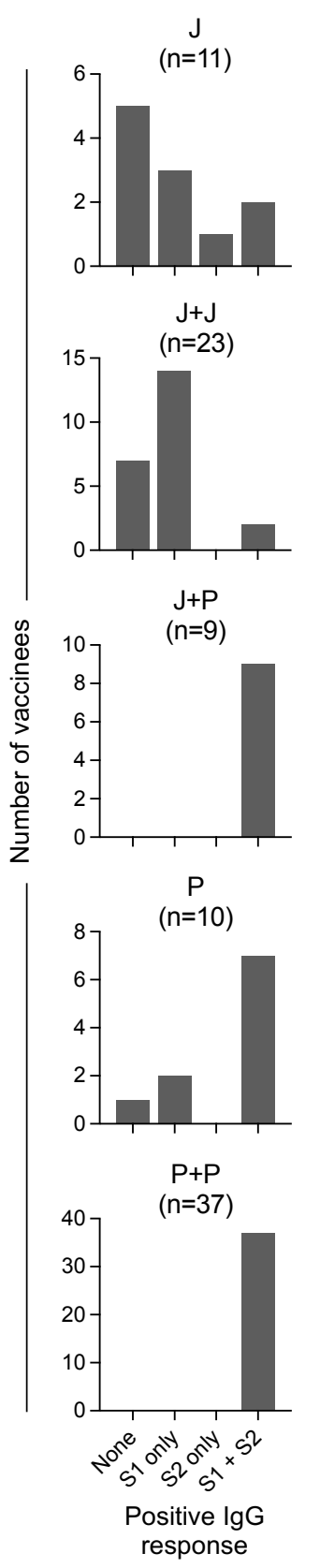

C
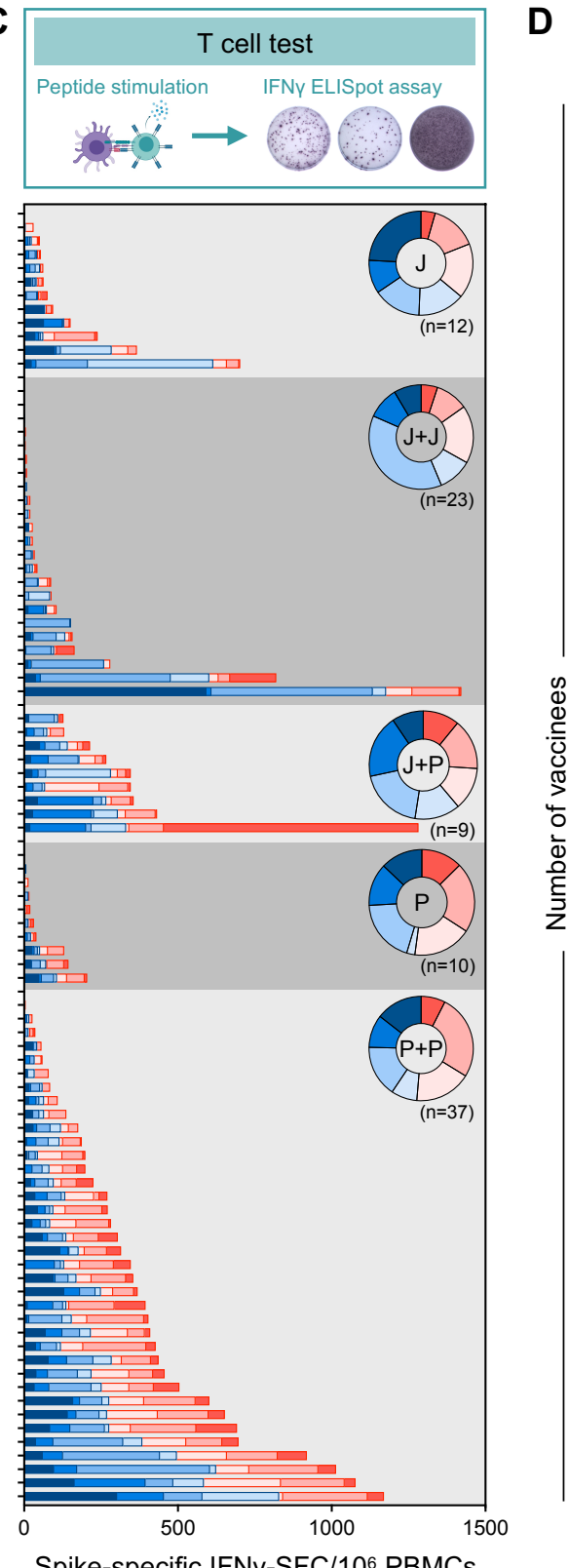

Spike-specific IFNY-SFC/106 PBMCs

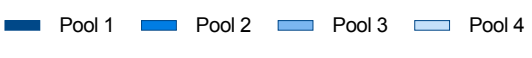

$\square$ Pool $5 \square$ Pool $6 \square$ Pool 7
D

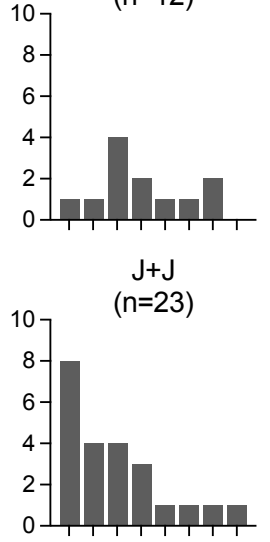

$J+P$

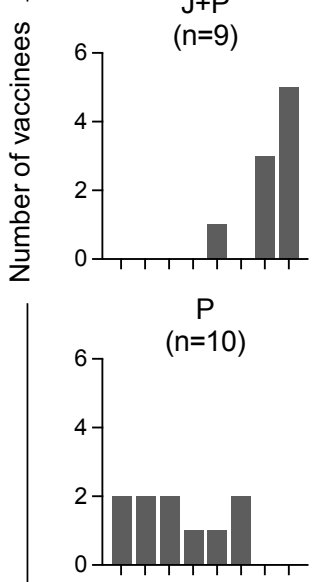

$\mathrm{P}+\mathrm{P}$

$(n=37)$

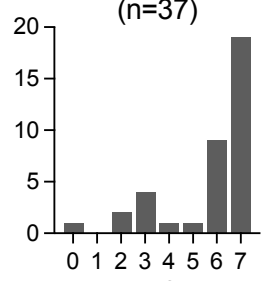

Number of positive spike peptide pools

\section{Figure 3: Qualitative profile of Spike-specific humoral and cellular immune responses.}

(A) Stacked bars represent IgG antibody titers against the S1 (blue) and S2 (pink) chains of SARS-CoV-2 Spike antigen. Each column represents an individual. Donut plots represent the mean of percentage of IgG antibodies against S1 or S2. (B) Frequency of with Spike-specific IgG antibodies recognizing none, S1, S2 or both S1 and S2 regions of Spike. IgG titers $>1.35 \mu \mathrm{g} / \mathrm{ml}$ were considered positive. (C) Stacked bars represent frequency of IFN- $\gamma$-spot forming cells (SFC) reactive to the individual Spike peptide pools (1 to 7) in each vaccinee. Donut chart represent the percentage mean of IFN- $\gamma$-SFC reactive to the individual Spike peptide pools. (D) Bar graphs show the frequency of vaccinees with varying breadth of Spike-specific $T$ cell response, determined by the number of positive Spike peptide pools. Responses $>7.5 \mathrm{SFC} / 10^{6}$ PBMCs were considered positive. 
A
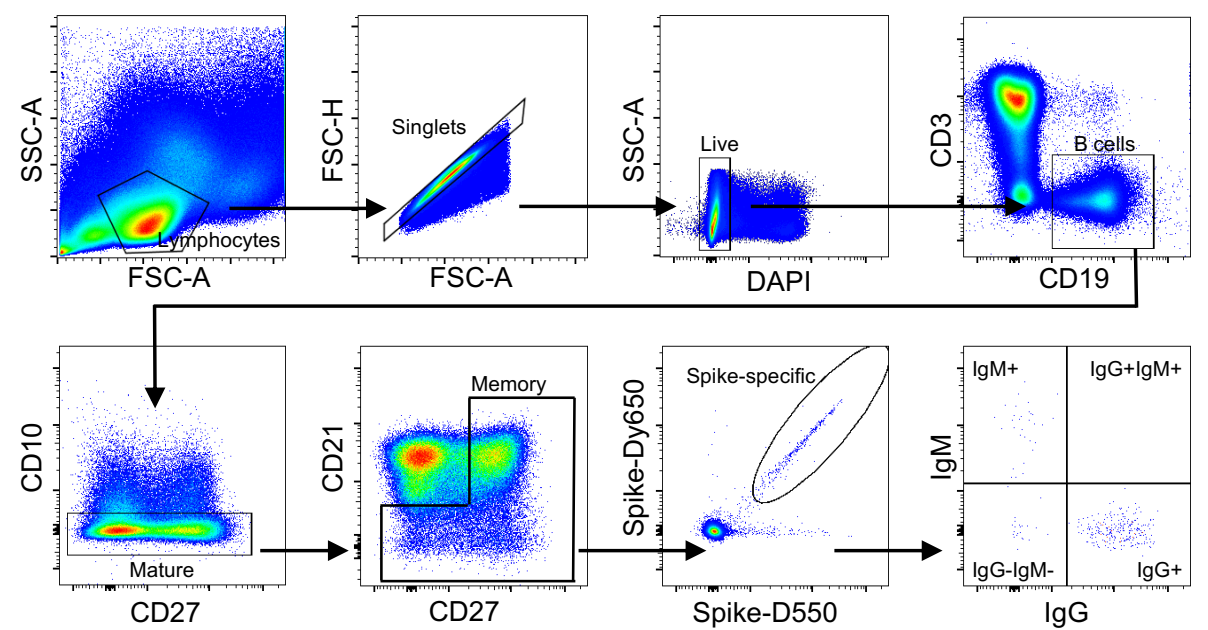

C

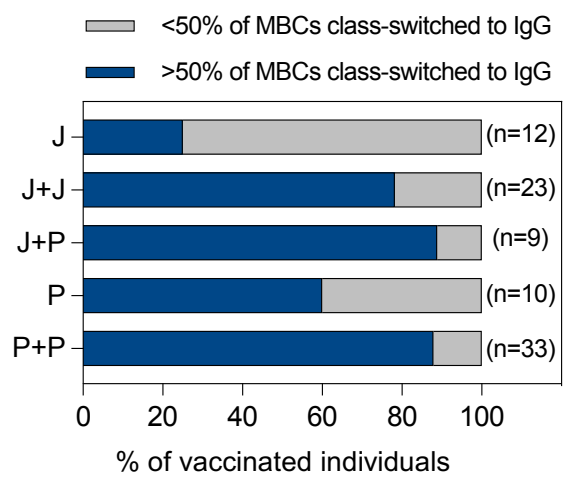

B

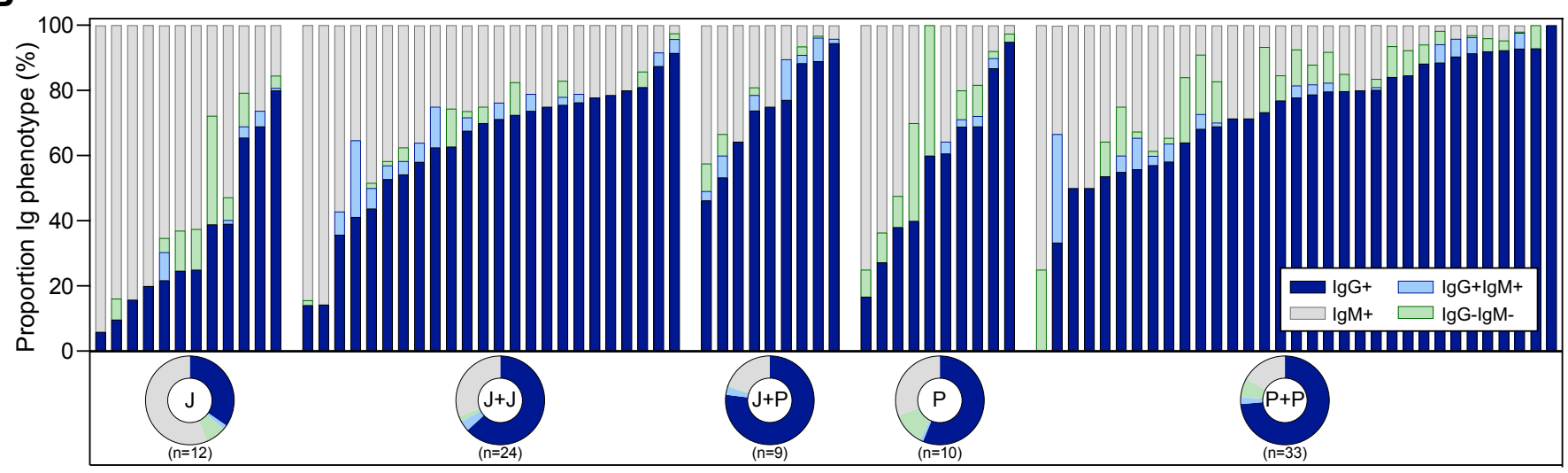

Figure 4: Phenotypic analysis of immunoglobulin isotypes of Spike-specific memory B cells

(A) Representative gating strategy of Spike-specific memory B cells (MBCs) expressing different immunoglobulin (Ig) types. (B) Stacked bars represent the frequency of indicated Ig isotypes expressed on Spike-specific MBCs. Corresponding donut plots represent the mean proportion of the four Ig categories (IgG+, IgG-IgM-, IgG+lgM+ and $\mathrm{IgM+}$. (C) Frequency of individuals with $>50 \%$ of MBCs class-switched to IgG (blue). 
se to display the preprint in perpetu

A Spike IgG (Log10[lgG])

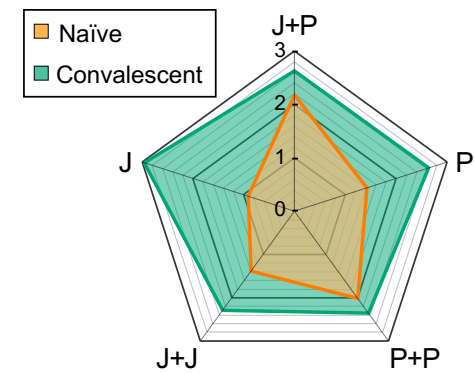

B

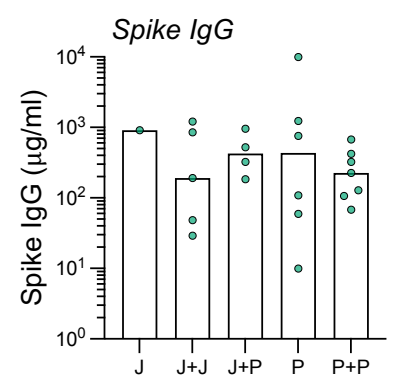

Neutralizing $A b$

(\% inhibition)

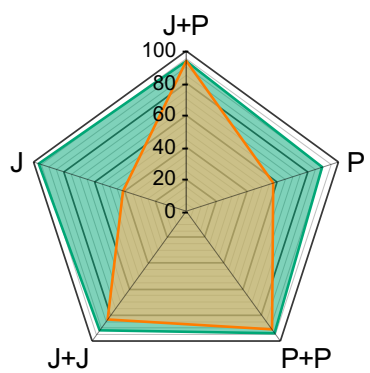

Neutralizing $A b$

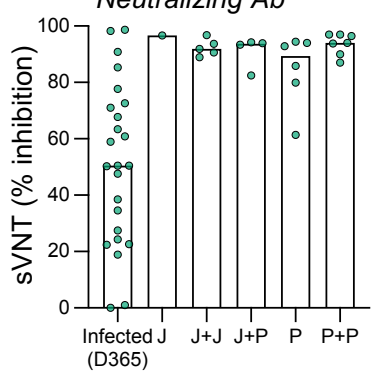

Memory B cells

(\% MBCs frequency)
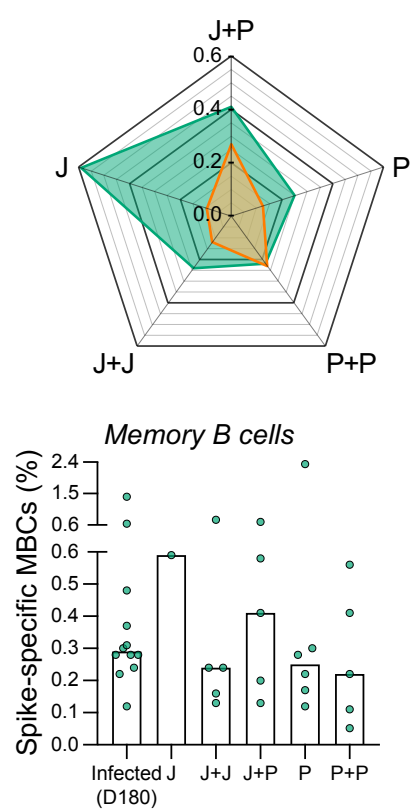

T cells

( $\log _{10}$ [IFNy SFC/106 PBMCs])
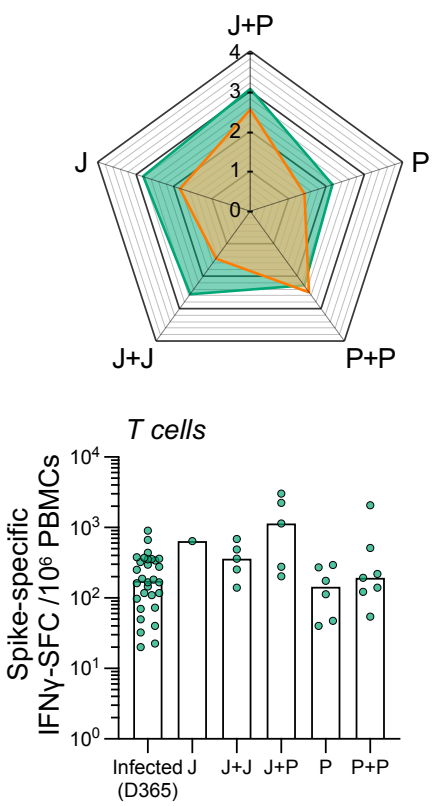

Figure 5: Spike-specific humoral and cellular immune response in convalescent vaccinated individuals.

Spike IgG antibody, sVNT, Spike-specific MBCs and T cell responses were tested in five cohorts of naïve vaccinated individuals: J $(n=12), J+J(n=23), J+P(n=9), P(n=10)$ and $P+P(n=37)$. Same analysis was carried out in SARS-CoV-2 convalescents vaccinated with: $J(n=1), J+J(n=5), P(n=6), P+P(n=7)$ and $J+P(n=5)$. A convalescent unvaccinated cohort was used as a control: Infected $(n=10-30)$. (A) The four radar plots show respectively median levels of Spike IgG antibody titers, neutralizing antibodies, frequency of Spike-specific memory B cells and T cells in convalescent (green; $n=24$ ) and naïve (orange; $n=87-91$ ) vaccinated individuals. Vaccination strategies $(J, J+J, J+P, P$ and $P+P)$ were indicated in the vertex of each pentagon. (B) Quantities of Spike IgG antibody, neutralizing antibodies, spike-specific memory B cells and T cells from convalescent vaccinated individuals. Bars denote the median value of each group. Each dot represents an individual. Significant differences in each group were analysed by one-way ANOVA and the adjusted $\mathrm{p}$-values (adjusted for multiple comparison) are shown. No significance is not shown; ${ }^{*}=\mathrm{P} \leq 0.05 ;{ }^{* *}=$ $\mathrm{P} \leq 0.01 ; * * *=\mathrm{P} \leq 0.001 ; * * * *=\mathrm{P} \leq 0.0001$. 


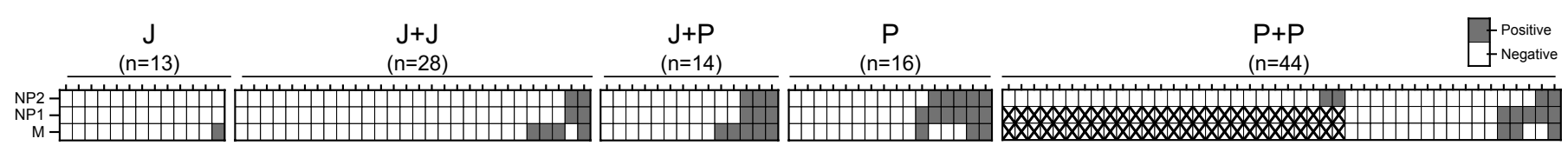

\section{Supplementary Figure 1: Identification of convalescent vaccinated individuals}

Heatmap shows the SARS-CoV-2 non-Spike-specific T cell responses quantified in vaccinated individuals using IFN- $\gamma$ ELISpot. Individuals with at least one positive response (>20 SFC SFC/106 PBMCs) are categorized as potentially SARSCoV-2 convalescent vaccinees. While individuals with no positive response are categorized as naïve vaccinees. " $X$ " denotes structural peptide pools that were untested. 


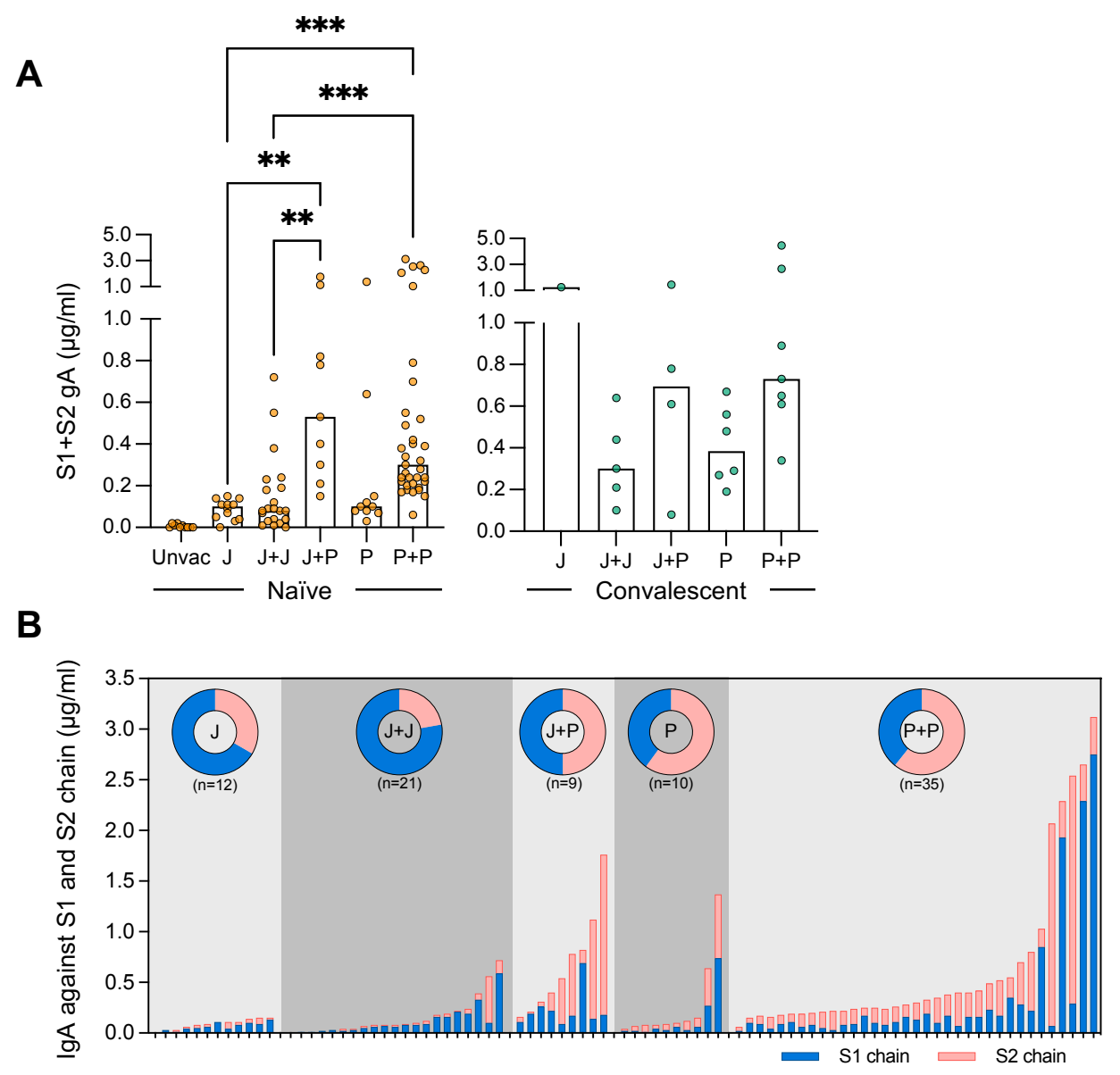

\section{Supplementary Figure 2: Quantitative and Qualitative profile of Spike-specific IgA antibodies}

(A) Spike-specific IgA antibody responses were tested in five cohorts of naïve vaccinated individuals: J $(n=12), J+J$ $(n=23), J+P(n=9), P(n=10)$ and $P+P(n=37)$. Same analysis was carried out in convalescent vaccinated individuals: J $(n=1), J+J(n=4), J+P(n=5), P(n=6)$ and $P+P(n=7)$. A naïve unvaccinated cohort was used as a control, Unvac $(n=10)$. Bars denote the median value of each group. Each dot represents an individual. Significant differences in each group were analysed by one-way ANOVA and the adjusted p-values (adjusted for multiple comparison) are shown. No significance is not shown, ${ }^{*}=\mathrm{P} \leq 0.05 ;{ }^{* *}=\mathrm{P} \leq 0.01 ;{ }^{* * *}=\mathrm{P} \leq 0.001$. (B) Stacked bars represent IgA antibody titers against the S1 (blue) and S2 (pink) chains of SARS-CoV-2 Spike antigen. Each column represents an individual. Donut plots represent the mean of percentage of IgA antibodies against S1 or S2. 
A
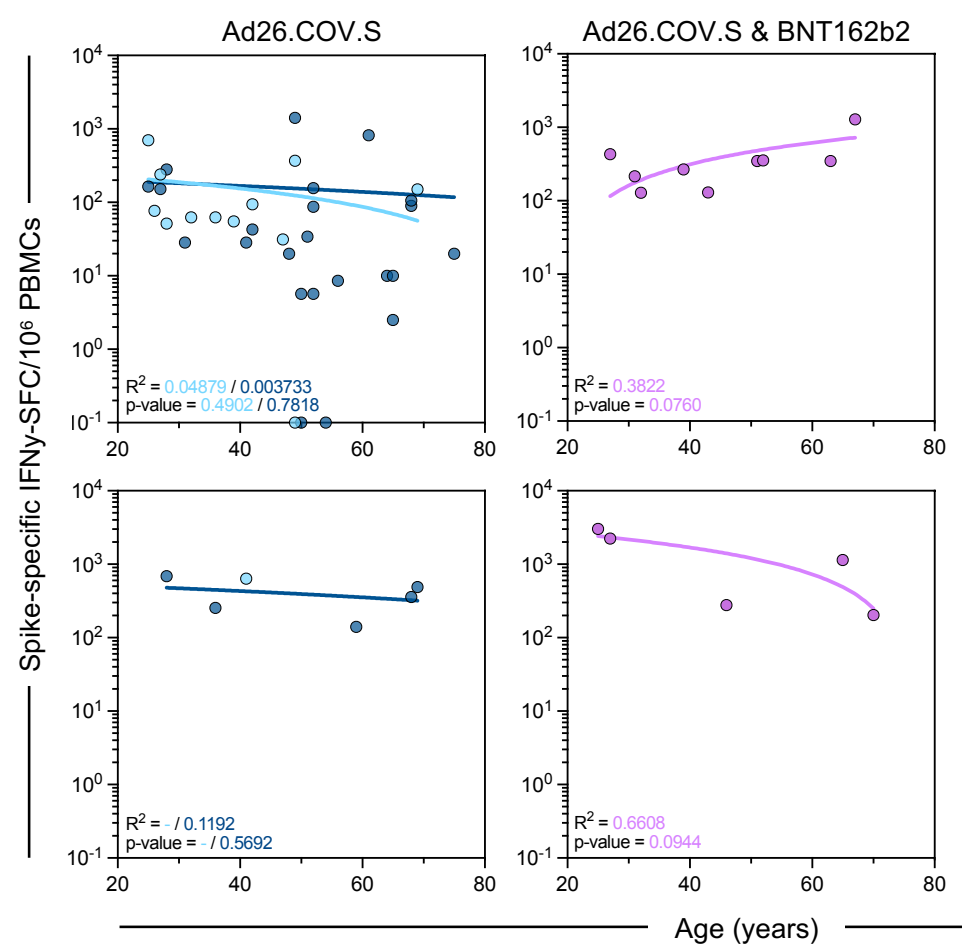

B

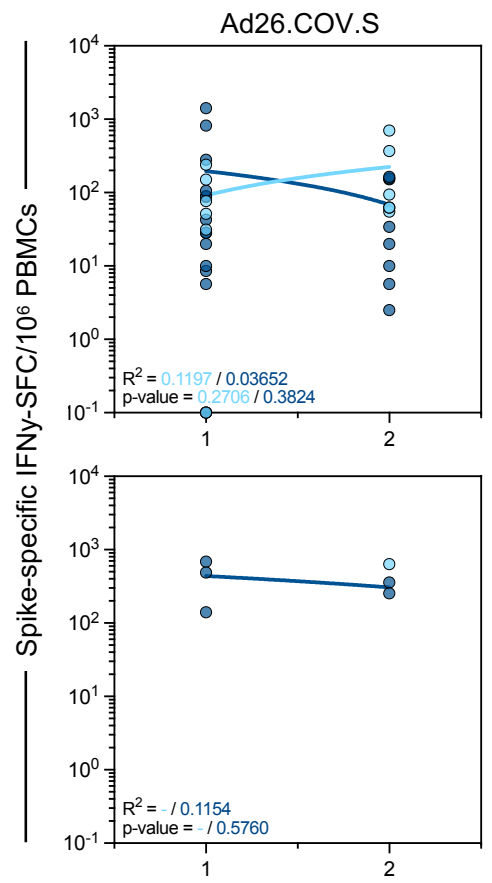

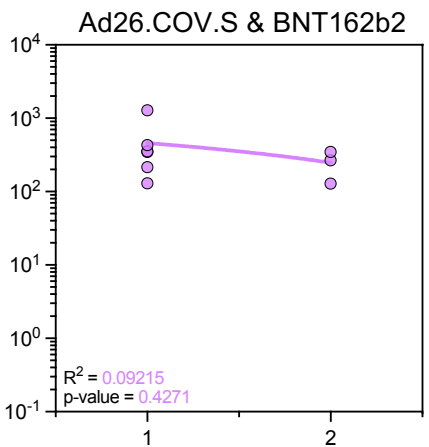

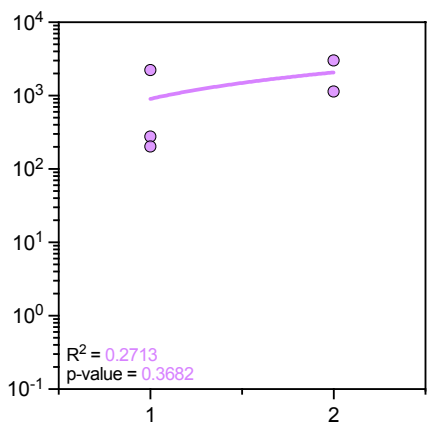

Sex $($ male $=1$, female $=2)$

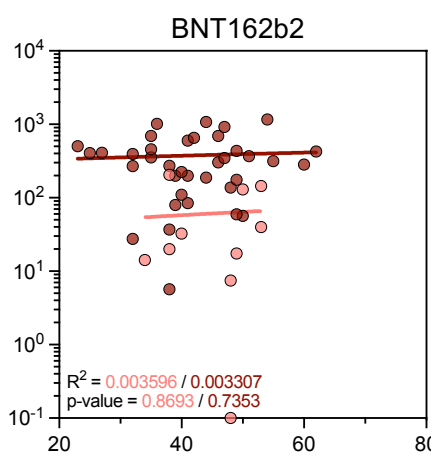

Naïve vaccinated

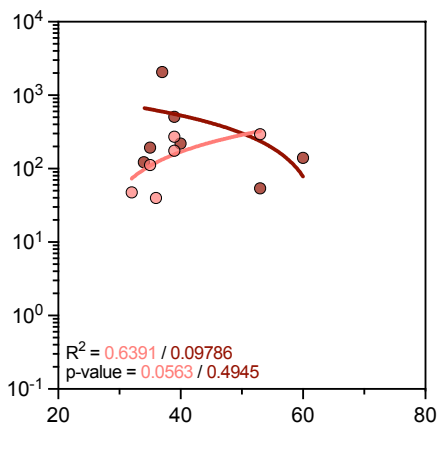

Convalescent vaccinated

Naïve

vaccinated

Convalescent vaccinated

\section{Supplementary Figure 3: Correlation of Spike-specific T cell frequency with age or sex}

Linear regression analysis between Spike-specific T cell frequency and (A) age or (B) sex of vaccine recipients who are naïve (top panel) or convalescents (bottom panel). Goodness of fit and p-value are shown in plots. 